\title{
Bifunctional Iminophosphorane Catalyzed Enantioselective Ketimine Phospha-Mannich Reaction
}

\author{
Gerard P. Robertson \\ Alistair J. M. Farley \\ Darren J. Dixon*
}

The Department of Chemistry, Chemistry Research Laboratory, University of Oxford, 12 Mansfield Road, Oxford OX1 3TA, UK

darren.dixon@chem.ox.ac.uk

Dedicated to Professor Steven V. Ley on the occasion of his $70^{\text {th }}$ birthday

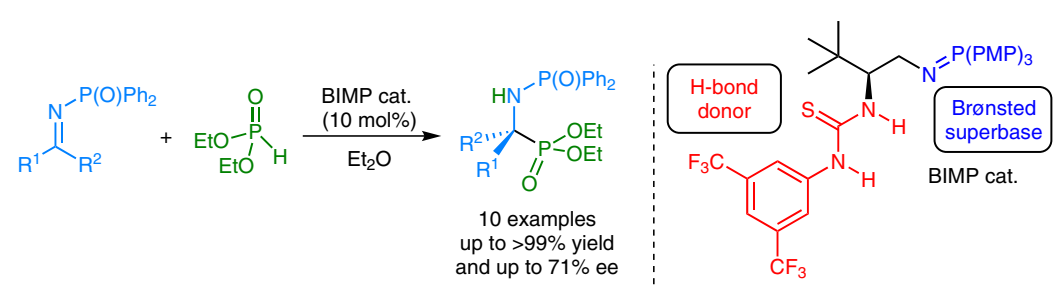

lyst-enabled substrate activation and enantioface discrimination; ${ }^{7}$ a problem that necessitates the use of metal ion catalysts, stoichiometric additives or the use of activated ketimine electrophiles. For example, Shibasaki and coworkers reported the highly enantioselective $N$-thiophosphinoyl ketimine phospha-Mannich reaction under copper(I) catalysis, ${ }^{8 a}$ whereas Nakamura et al. used catalytic quantities of commercially available cinchona alkaloids in the presence of super stoichiometric quantities of $\mathrm{Na}_{2} \mathrm{CO}_{3}$, for the enantioselective addition of diethyl phosphite to $\mathrm{N}$ mesitylene sulfonyl protected ketimines. ${ }^{8 b}$ Very recently, Chimni and co-workers and Reddy and co-workers described the catalytic enantioselective phospha-Mannich reaction of reactive isatin-derived ketimines catalyzed by bifunctional cinchona-derived thiourea and squaramide catalysts, respectively. ${ }^{8 c, d}$

In an attempt to overcome the reactivity problem of certain classes of electrophiles and pro-nucleophiles, we recently developed a new class of bifunctional superbase organocatalysts incorporating for the first time the triaryliminophosphorane moiety as the Brønsted base and with it achieved the first general enantioselective organocatalytic ketimine nitro-Mannich reaction. ${ }^{9}$ The juxtaposition of both the organosuperbase ${ }^{10}$ and an appropriate hydrogen bond donor group over a chiral scaffold was critical for successful enantioselective catalysis (high reactivity and enantiocontrol). As a part of this program into the development of novel asymmetric methodologies for challenging electrophiles, we wish to report the first organocatalytic enantioselective phospha-Mannich reaction of unactivated $N$-DPP ketimines.

We chose the 1,2-addition of diethyl phosphite 3 to the unactivated $N$-DPP-protected ketimine derived from acetophenone $\mathbf{2 a}$ as our model system for testing the performance of our bifunctional iminophosphorane (BIMP) catalysts (Table 1 and Figure 1). Promising reactivity was initial- 
ly established using $10 \mathrm{~mol} \%$ of our previously reported first-generation tert-leucine derived BIMP catalyst 1a with triphenylphosphine (Table 1, entry 1). After just 24 hours at room temperature, $74 \%$ yield of product $\mathbf{4 a}$ was afforded with an encouraging ee of $56 \%$. However, switching to the analogous but more basic catalyst $\mathbf{1 b}$ derived from tris( $p$ methoxyphenyl)phosphine gave rise to a significant boost in reactivity and a slight boost in enantiocontrol; adduct 4a was afforded in quantitative yield and with $58 \%$ ee (Table 1 , entry 2). The analogous L-phenylalanine or L-valine-derived catalysts, $1 \mathbf{c}$ and $\mathbf{1 d}$ respectively, resulted in a drop in enantioselectivity in both cases (Table 1, entries 3 and 4). Employing catalyst 1e, possessing the diphenylmethyl group as part of its chiral scaffold, resulted in a drop in the enantioselectivity to $23 \%$ ee (Table 1 , entry 5 ). Simple modification of the thiourea hydrogen bond donor group of the first-generation BIMP organocatalysts led to no improvement in the level of enantiocontrol (Table 1, entries 6 and 7), and accordingly alternative second-generation BIMP organocatalyst designs were considered. Introducing an additional amino acid residue ${ }^{11}$ between the iminophosphorane moiety and Schreiner-type thiourea ${ }^{12}$ allowed diastereomers $\mathbf{1 h}$ and $\mathbf{1 j}$ to be synthesized and compared in the reaction. Interestingly neither catalyst outperformed $\mathbf{1 b}$, but taken together showed that the valine residue in both catalysts was dominating enantioselectivity (Table 1, entries 8 and 10). Building on these observations catalyst $1 \mathbf{i}$ was tested in

Table 1 Proof of Concept and Optimization Studies in the Ketimine Phospha-Mannich Reaction ${ }^{\mathrm{a}}$

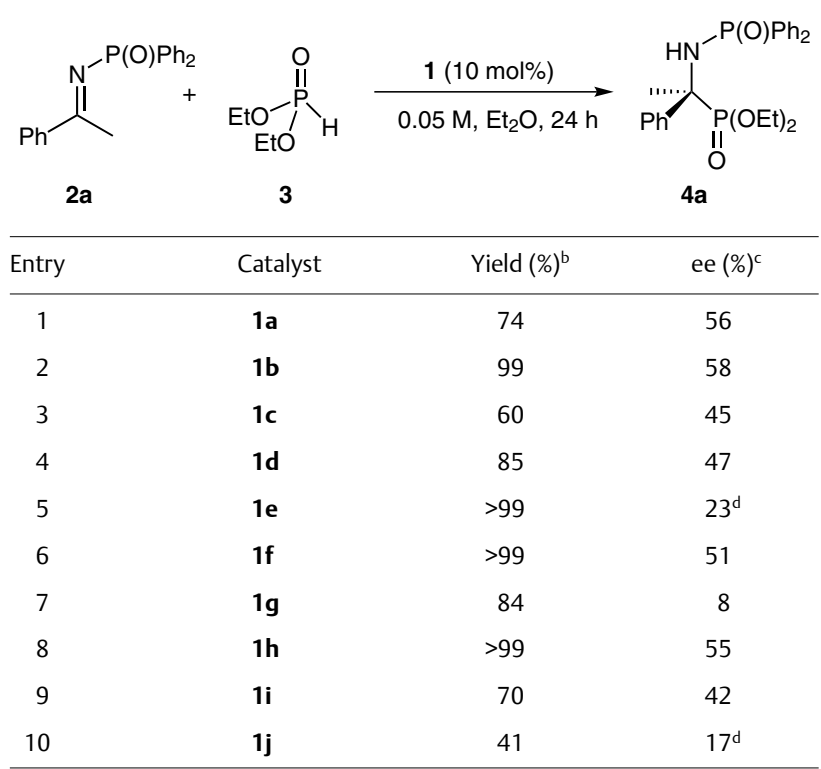

a Reactions were performed using $\mathbf{3}(0.20 \mathrm{mmol}), \mathbf{2 a}$ (2.0 equiv) and catalyst (10 mol\%).

${ }^{\mathrm{b}}$ Isolated yield.

c Determined by HPLC analysis on a chiral stationary phase.

${ }^{\mathrm{d}}$ Enantiomer $(R)-4$ a was obtained.

the reaction in the hope that an additional boost in selectivity would be witnessed, but disappointingly enantioselectivity was reduced to $42 \%$ ee (Table 1 , entry 9 ). Having identified the best catalyst as $\mathbf{1 b}$, a brief re-optimization of the reaction conditions, with respect to solvent, concentration and temperature, was carried out but no augmentation of the enantioselectivity was observed and the optimal conditions remained the same as for Table 1 , entry $2 .{ }^{13}$<smiles>FC(F)(F)c1cc(NC(=S)NC([Tl])CN=P)cc(C(F)(F)F)c1</smiles>

1a: $R^{1}=t-B u, R^{2}=P h$

1b: $R^{1}=t-B u, R^{2}=P M P$

1c: $R^{1}=B n, R^{2}=P M P$

1d: $\mathrm{R}^{1}=i-\operatorname{Pr}, \mathrm{R}^{2}=\mathrm{PMP}$<smiles>CONC(CN=[Pb])C(C)(C)C</smiles>

1f: $\mathrm{EWG}=\mathrm{C}(\mathrm{O}) \mathrm{NH}\left(m, m-\left(\mathrm{CF}_{3}\right) \mathrm{C}_{6} \mathrm{H}_{3}\right)$ 1g: $\mathrm{EWG}=\mathrm{C}(\mathrm{O})\left(m, m-\left(\mathrm{CF}_{3}\right)_{2} \mathrm{C}_{6} \mathrm{H}_{3}\right)$ $\mathrm{PMP}=$ para-methoxyphenyl

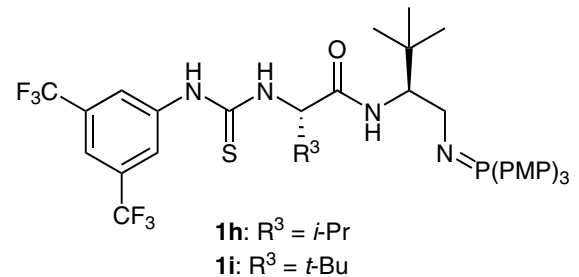<smiles>CCCCCCC[P+](C)=NCC(NC(=O)C(NC(=S)Nc1cc(C(F)(F)F)cc(C(F)(F)F)c1)C(C)C)C(C)(C)C</smiles>

Figure 1 First- and second-generation BIMP catalysts

With optimized conditions in hand, we next investigated the substrate scope and found good tolerance over a range of electron-rich and electron-deficient aromatic ketimines: yields were typically $>99 \%$ and enantioselectivities ranged from 41 to $62 \%$ ee (Scheme 1). Furthermore, a 3-pyridyl substrate performed well ( $>99 \%$ yield, $53 \%$ ee) and pleasingly the reaction was also applicable to the ethyl homologue of $\mathbf{2 a}$ which afforded product $\mathbf{4} \mathbf{j}$ in $\mathbf{7 1 \%}$ ee and in quantitative yield. Absolute configuration of $\mathbf{4 a}$ was established as $S$ by comparison of the specific rotation of a derivative with that of a literature compound (see Supporting Information). 


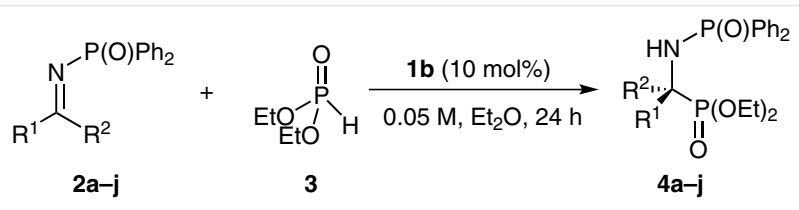

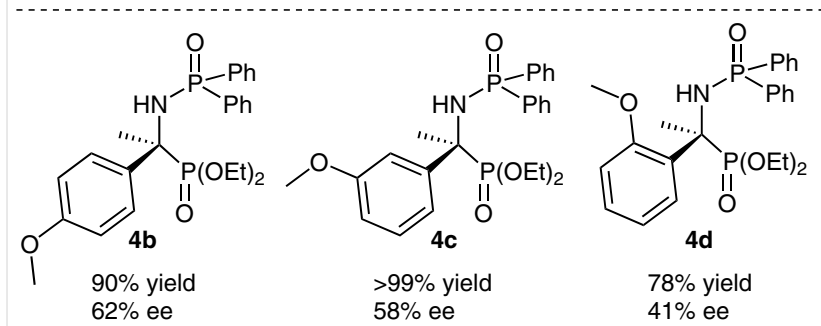<smiles>CCOC(=O)C(NP(=O)(c1ccccc1)c1ccccc1)c1ccc(Cl)cc1</smiles><smiles>CCOC(=O)[C@](C)(NP(=O)(c1ccccc1)c1ccccc1)c1ccc(Br)cc1</smiles><smiles>CCOP(=O)(OCC)[C@](C)(NP(=O)(c1ccccc1)c1ccccc1)c1ccc(F)cc1</smiles><smiles>CCO[P+](=O)C(C)(NP(=O)(c1ccccc1)c1ccccc1)c1cccnc1</smiles><smiles>CCO[P+](=O)C(C)(NP(=O)(c1ccccc1)c1ccccc1)c1cc(C(F)(F)F)cc(C(F)(F)F)c1</smiles>

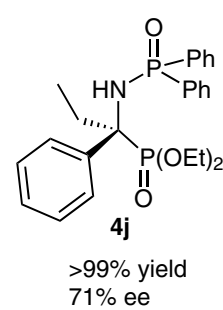

Scheme 1 Scope of the BIMP-catalyzed phospha-Mannich reaction ${ }^{\mathrm{a}}$

In summary we have developed an organocatalytic ketimine phospha-Mannich reaction of diethyl phosphite to unactivated $N$-DPP ketimines with excellent yields and moderate enantioselectivities. Further work focussing on the development of novel asymmetric methodologies for challenging electrophiles is ongoing in our group and the results will be disclosed in due course.

\section{Acknowledgment}

This work was supported by the EPSRC (Studentship to G.P.R. and A.J.M.F. and Doctoral Prize [EP/M50659X/1] to A.J.M.F.), AstraZeneca (Studentship to A.J.M.F.) and the SCI (Postgraduate Scholarship to A.J.M.F.)

\section{Supporting Information}

Supporting information for this article is available online at http://dx.doi.org/10.1055/s-0035-1560530.

\section{References and Notes}

(1) (a) Hiratake, J.; Oda, J. Biosci., Biotechnol., Biochem. 1997, 61, 211. (b) Aminophosphonic and Aminophosphinic Acids; Kukhar, V. P.; Hudson, H. R., Eds.; John Wiley \& Sons: New York, 2000.

(2) (a) Allen, J. G.; Atherton, F. R.; Hall, M. J.; Hassal, C. H.; Holmes, S. W.; Lambert, R. W.; Nisbet, L. J.; Ringrose, P. S. Nature (London) 1978, 272, 56. (b) Atherton, F. R.; Hassall, C. H.; Lambert, R. W.J. Med. Chem. 1986, 29, 29. (c) Stowasser, B.; Budt, K.-H.; JianQi, L.; Peyman, A.; Ruppert, D. Tetrahedron Lett. 1992, 33, 6625. (d) Alonso, E.; Solis, A.; del Pozo, C. Synlett 2000, 698. (e) Hirschmann, R.; Smith, A. B. III.; Taylor, C. M.; Benkovic, P. A.; Taylor, S. D.; Yager, K. M.; Sprengeler, P. A.; Benkovic, S. J. Science 1994, 265, 234. (f) Smith, W. W.; Bartlett, P. A. J. Am. Chem. Soc. 1998, 120,4622 .

(3) (a) Enders, D.; Saint-Dizier, A.; Lannou, M.-I.; Lenzen, A. Eur. J. Org. Chem. 2006, 29. (b) Ordóñez, M.; Rojas-Cabrera, H.; Cativiela, C. Tetrahedron 2009, 65, 17. (c) Albrecht, L.; Albrecht, A.; Krawczyk, H.; Jørgensen, K. A. Chem. Eur. J. 2010, 16, 28. (d) Bera, K.; Namboothiri, I. N. N. Asian J. Org. Chem. 2014, 3, 1234. (e) Kolodiazhnyi, O. I.; Kukhar, V. P.; Kolodiazhna, A. O. Tetrahedron: Asymmetry 2014, 25, 865. (f) Faisca Phillips, A. M. Mini-Rev. Org. Chem. 2014, 11, 164. (g) Dziegielewski, M.; Pieta, J.; Kaminska, E.; Albrecht, L. Eur. J. Org. Chem. 2015, 677.

(4) (a) Merino, P.; Marqués-López, E.; Herrera, R. P. Adv. Synth. Catal. 2008, 350, 1195. (b) Angelini, T.; Bonollo, S.; Lanari, D.; Pizzo, F.; Vaccaro, L. Org. Biomol. Chem. 2013, 11, 5042.

(5) For reviews on organocatalysis, see: (a) Dalko, P. I.; Moisan, L. Angew. Chem. Int. Ed. 2004, 43, 5138. (b) Berkessel, A.; Gröger, H. Asymmetric Organocatalysis; Wiley-VCH: Weinheim, 2005. (c) List, B.; Yang, J. W. Science 2006, 313, 1584. (d) Dondoni, A.; Massi, A. Angew. Chem. Int. Ed. 2008, 47, 4638. (e) Bertelsen, S.; Jørgensen, K. A. Chem. Soc. Rev. 2009, 38, 2178. (f) Palomo, C.; Oiarbide, M.; López, R. Chem. Soc. Rev. 2009, 38, 632. For reviews on Brønsted base/H-bond donor bifunctional organocatalysts, see: (g) Takemoto, Y. Org. Biomol. Chem. 2005, 3, 4299. (h) Connon, S. J. Chem. Commun. 2008, 2499. (i) Marcelli, T.; Hiemstra, H. Synthesis 2010, 1229. (j) Quintavella, A.; Cerisoli, L.; Montroni, E. Current Organocatalysis 2014, 1, 107.

(6) For selected examples using metals, see: (a) Gröger, H.; Saida, Y.; Sasai, H.; Yamaguchi, K.; Martens, J.; Shibasaki, M. J. Am. Chem. Soc. 1998, 120, 3089. (b) Abell, J. P.; Yamamoto, H. J. Am. Chem. Soc. 2008, 130, 10521. (c) Huang, M.; Li, C.; Huang, J.; Duan, W.-L.; Xu, S. Chem. Commun. 2012, 48, 11148. For selected examples using organocatalysts, see: (d) Pettersen, D.; Marcolini, M.; Bernardi, L.; Fini, F.; Herrera, R. P.; Sgarzani, V.; Ricci, A. J. Org. Chem. 2006, 71, 6269. (e) Joly, G. D.; Jacobsen, E. N. J. Am. Chem. Soc. 2004, 126, 4102. (f) Akiyama, T.; Morita, H.; Itoh, J.; Fuchibe, K. Org. Lett. 2005, 7, 2583. (g) Nakamura, S.; Nakashima, H.; Sugimoto, H.; Sano, H.; Hattori, M.; Shibata, N.; Toru, T. Chem. Eur. J. 2008, 14, 2145. (h) Fu, X.; Loh, W.-T.; Zhang, Y.; Chen, T.; Ma, T.; Liu, H.; Wang, J.; Tan, C.-H. Angew. Chem. Int. Ed. 2009, 48, 7387.

(7) (a) Riant, O.; Hannedouche, J. Org. Biomol. Chem. 2007, 5, 873. (b) Shibasaki, M.; Kanai, M. Chem. Rev. 2008, 108, 2853.

(8) (a) Yin, L.; Bao, Y.; Kumagai, N.; Shibasaki, M. J. Am. Chem. Soc. 2013, 135, 10338. (b) Nakamura, S.; Hayashi, M.; Hiramatsu, Y.; Shibata, N.; Funahashi, Y.; Toru, T. J. Am. Chem. Soc. 2009, 131, 18240. (c) Kumar, A.; Sharma, V.; Kaur, J.; Kumar, V.; Mahajan, S.; Kumar, N.; Chimni, S. S. Tetrahedron 2014, 70, 7044. (d) George, J.; Sridhar, B.; Reddy, B. V. S. Org. Biomol. Chem. 2014, 12, 1595. 
(9) (a) Núñez, M. G.; Farley, A. J. M.; Dixon, D. J. J. Am. Chem. Soc. 2013, 135, 16348. (b) Goldys, A. M.; Núñez, M. G.; Dixon, D. J. Org. Lett. 2014, 16, 6294. (c) Goldys, A. M.; Dixon, D. J. Macromolecules 2014, 47, 1277.

(10) (a) Ishikawa, T. Superbases for Organic Synthesis: Guanidines, Amidines, Phosphazenes and Related Organocatalysts; Wiley: New York, 2009. For a review on chiral organosuperbases, see: (b) Ishikawa, T.; Kumamoto, T. Synthesis 2006, 737. (c) Leow, D.; Tan, C.-H. Chem. Asian J. 2009, 4, 488. (d) Leow, D.; Tan, C.-H. Synlett 2010, 1589. (e) Ishikawa, T. Chem. Pharm. Bull. 2010, 58, 1555. (f) Fu, X.; Tan, C.-H. Chem. Commun. 2011, 47, 8210. (g) Selig, P. Synthesis 2013, 45, 703. (h) Krawczyk, H.; Dzięgielewski, M.; Deredas, D.; Albrecht, A.; Albrecht, Ł. Chem. Eur. J. 2015, 21, 10268. For recent selected examples, see: (i) Nugent, B. M.; Yoder, R. A.; Johnston, J. N. J. Am. Chem. Soc. 2004, 126, 3418. (j) Terada, M.; Ube, H.; Yaguchi, Y. J. Am. Chem. Soc. 2006, 128, 1454. (k) Uraguchi, D.; Sakaki, S.; Ooi, T. J. Am. Chem. Soc. 2007, 129, 12392. (l) Sohtome, Y.; Shin, B.; Horitsugi, N.; Takagi, R.; Noguchi, K.; Nagasawa, K. Angew. Chem. Int. Ed. 2010, 49, 7299. (m) Fu, X.; Tan, C.-H. Chem. Commun. 2011, 47, 8210. (n) Bandar, J. S.; Lambert, T. H. J. Am. Chem. Soc. 2012, 134, 5552. (o) Takeda, T.; Terada, M. J. Am. Chem. Soc. 2013, 135, 15306. (p) Issik, M.; Unver, M. Y.; Tanyeli, C. J. Org. Chem. 2015, 80, 828. (q) Gao, X.; Han, J.; Wang, L. Org. Lett. 2015, 17, 4596.
(11) Zhu, Q. A.; Lu, Y. X. Angew. Chem. Int. Ed. 2010, 49, 7753.

(12) Wittkopp, A.; Schreiner, P. R. Chem. Eur. J. 2003, 9, 407.

(13) Representative Procedure for the Enantioselective Ketimine Phospha-Mannich Reaction: To a solution of ketimine 2a (128 $\mathrm{mg}, 0.40 \mathrm{mmol}, 2.0$ equiv) and catalyst $\mathbf{1 b}(15 \mathrm{mg}, 0.020 \mathrm{mmol}$, 0.10 equiv in $4.0 \mathrm{~mL}$ of diethyl ether) was added diethyl phosphite 3 ( $26 \mu \mathrm{L}, 0.20 \mathrm{mmol}, 1.0$ equiv) at rt. Stirring was maintained for $24 \mathrm{~h}$ whereupon the crude reaction mixture was purified directly by flash column chromatography [petroleum ether to petroleum ether-EtOAc (1:2), EtOAc then EtOAc-MeOH (9:1)] to afford the phospha-Mannich addition product 4 a.

Diethyl \{(1S)-1-[(Diphenylphosphoryl)amino]-1-phenylethyl\}phosphonate (4a): The title compound $\mathbf{4 a}$ was isolated in $99 \%$ yield (91 mg) and 58\% ee as a colorless solid. ${ }^{1} \mathrm{H}$ NMR $(500 \mathrm{MHz}$, $\left.\mathrm{CDCl}_{3}\right): \delta=1.03(\mathrm{t}, J=7.0 \mathrm{~Hz}, 3 \mathrm{H}), 1.25(\mathrm{t}, J=7.0 \mathrm{~Hz}, 3 \mathrm{H}), 1.82$ $\left(\mathrm{d}, J_{\mathrm{PH}}=17.0 \mathrm{~Hz}, 3 \mathrm{H}\right), 3.53(\mathrm{ddq}, J=10.0,7.0,7.0 \mathrm{~Hz}, 1 \mathrm{H}), 3.81$ (ddq, $J=10.0,7.0,7.0 \mathrm{~Hz}, 1 \mathrm{H}), 4.03-4.20(\mathrm{~m}, 3 \mathrm{H}), 7.18-7.30$ (m, $5 \mathrm{H}), 7.31-7.47(\mathrm{~m}, 4 \mathrm{H}), 7.51(\mathrm{dd}, J=7.5,1.5 \mathrm{~Hz}, 2 \mathrm{H}), 7.55-7.64$ (m, $2 \mathrm{H}), 7.82-7.91(\mathrm{~m}, 2 \mathrm{H}) .{ }^{31} \mathrm{P}$ NMR $\left(162 \mathrm{MHz}, \mathrm{CDCl}_{3}\right): \delta=$ $20.1\left(J_{\mathrm{PP}}=29.3 \mathrm{~Hz}\right), 24.8\left(J_{\mathrm{PP}}=29.3 \mathrm{~Hz}\right)$. HRMS: $\left(\mathrm{ESI}^{+}\right): \mathrm{m} / z$ calcd for $\mathrm{C}_{24} \mathrm{H}_{29} \mathrm{NNaO}_{4} \mathrm{P}_{2}$ : 480.1464; found: 480.1454 . See Supporting Information for full characterization data. 